NASA Contractor Report 189692

ICASE Report No. 92-39
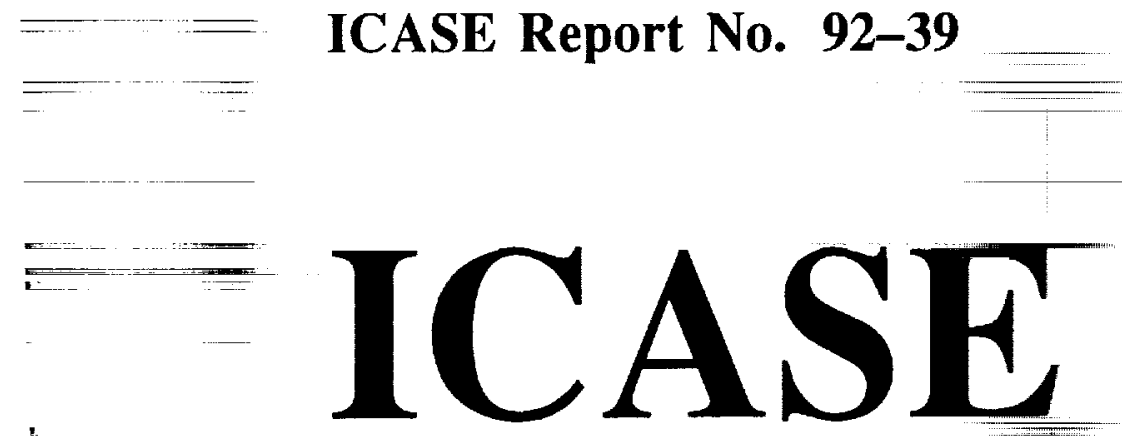

\title{
A NUMERICAL RESOLUTION STUDY OF HIGH ORDER ESSENTIALLY NON-OSCILLATORY SCHEMES APPLIED TO INCOMPRESSIBLE FLOW
}

\section{Weinan $\mathbf{E}$ \\ Chi-Wang Shu}

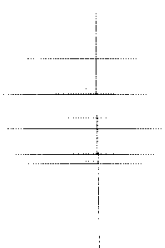

Contract Nos. NAS1-18605 and NAS1-19480

August 1992

Institute for Computer Applications in Science and Engineering NASA Langley Research Center

Hampton, Virginia 23665-5225

Operated by the Universities Space Research Association

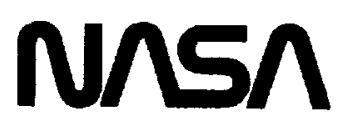

National Aeronautics and

Space Administration

Langley Research Center

Hamplon, Virginia 23665-5225

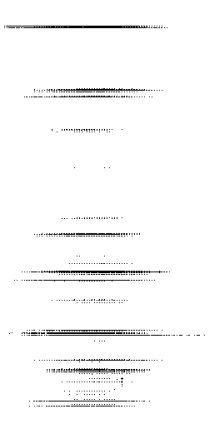

$\begin{array}{lll}0 & & \\ m & n & n \\ m & 0 & 0 \\ 1 & \frac{1}{N} & 0 \\ a & 5 & n \\ z & 5 & 0\end{array}$

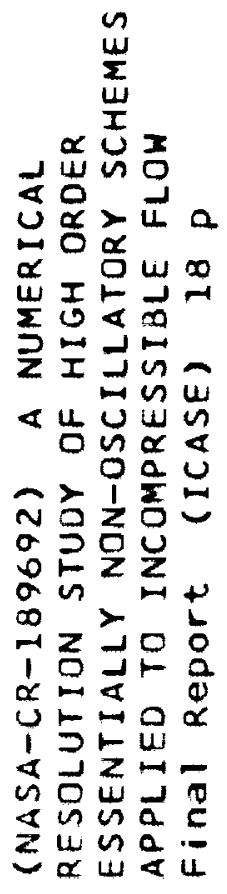




\title{
A NUMERICAL RESOLUTION STUDY OF HIGH ORDER ESSENTIALLY NON-OSCILLATORY SCHEMES APPLIED TO INCOMPRESSIBLE FLOW ${ }^{1}$
}

\author{
Weinan $E^{2}$ \\ School of Mathematics \\ Institute for Advanced Study \\ Princeton, New Jersey 08540 \\ and \\ Chi-Wang Shu ${ }^{3}$ \\ Division of Applied Mathematics \\ Brown University \\ Providence, Rhode Island 02912
}

\begin{abstract}
High order essentially non-oscillatory (ENO) schemes, originally designed for compressible flow and in general for hyperbolic conservation laws, are applied to incompressible Euler and Navier-Stokes equations with periodic boundary conditions. The projection to divergence-free velocity fields is achieved by fourth order central differences through Fast Fourier Transforms (FFT) and a mild high-order filtering. The objective of this work is to assess the resolution of ENO schemes for large scale features of the flow when a coarse grid is used and small scale features of the flow, such as shears and roll-ups, are not fully resolved. It is found that high-order ENO schemes remain stable under such situations and quantities related to large-scale features, such as the total circulation around the roll-up region, are adequately resolved.
\end{abstract}

\footnotetext{
${ }^{1}$ Computation supported by Pittsburgh Supercomputer Center.

${ }^{2}$ Research supported by AFOSR grant $90-0090$ and NSF grant DMS-9100383.

${ }^{3}$ Research supported by ARO grant DAAL03-91-G-0123, NASA Langley grant NAG1-1145 and NASA Langley contract NAS1-18605.
} 


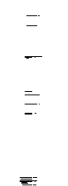

$=$

$=$

$\overline{\bar{\equiv}}$ 


\section{Introduction}

In this paper we consider numerically solving the incompressible Navier-Stokes or Euler equations

$$
\begin{aligned}
u_{t}+u u_{x}+v u_{y} & =\mu\left(u_{x x}+u_{y y}\right)-p_{x} \\
v_{t}+u v_{x}+v v_{y} & =\mu\left(v_{x x}+v_{y y}\right)-p_{y} \\
u_{x}+v_{y} & =0
\end{aligned}
$$

or their equivalent conservative form

$$
\begin{aligned}
u_{t}+\left(u^{2}\right)_{x}+(u v)_{y} & =\mu\left(u_{x x}+u_{y y}\right)-p_{x} \\
v_{t}+(u v)_{x}+\left(v^{2}\right)_{y} & =\mu\left(v_{x x}+v_{y y}\right)-p_{y} \\
u_{x}+v_{y} & =0
\end{aligned}
$$

where $(u, v)$ is the velocity vector, $p$ is the pressure, $\mu>0$ for the Navier-Stokes equations and $\mu=0$ for the Euler equations. The numerical methods we use are the high-order essentially non-oscillatory (ENO) schemes, originally designed for compressible flow and in general for hyperbolic conservation laws [8], [12]. The equation is defined on the box $[0,2 \pi] \times[0,2 \pi]$ with periodic boundary conditions in both directions. We choose two space dimensions for easy presentation, although our method is also applicable for three space dimensions.

In some sense equations (1.1) are easier to solve numerically than their compressible counter-parts because the latter have solutions containing possible discontinuities (for example shocks and contact discontinuities). However, the solution to (1.1), even if for most cases smooth mathematically, may evolve rather rapidly with time $t$ and may easily become too complicated to be fully resolved on a feasible grid. Traditional linearly stable schemes, such as spectral methods and high-order central difference methods, are suitable for the cases where the solution can be fully resolved, but typically produce signs of instability such as oscillations when small scale features of the flow, such as shears and roll-ups, cannot be adequately resolved on the computational grid (see, for example, the last contour in Fig. 1). Although in principle one can always overcome this difficulty by refining the grid, today's computer capacity seriously restricts the largest possible grid size.

In the last few years there is a lot of activity in designing high-order, nonlinearly stable "shock capturing" schemes for compressible flow and in general for hyperbolic conservation laws. See, for example, [2], [7], [8], [12], and the references listed therein. The philosophy of such schemes is to give up fully resolving rapid transition regions or shocks, just to "capture" 
them in a stable and somehow globally correct fashion (e.g., with correct shock speed), but at the same time to require a high resolution for the smooth part of the flow. The success of such an approach for the compressible flow is documented by many examples in the literature. One example is the one and two dimensional shock interaction with vorticity or entropy waves [12], [13]. The shock is captured sharply and certain key quantities related to the interaction between the shock and the smooth part of the flow, such as the amplification and generation factors when a wave passes through a shock, are well resolved. Another example is the homogeneous turbulence for compressible Navier-Stokes equations studied in [13]. In one of the test cases, the spectral method can resolve all the scales using a $256^{2}$ grid, while third order ENO with just $64^{2}$ points can adequately resolve certain interesting quantities such as the average fluctuation Mach number and maximum divergence, although it cannot resolve local quantities achieved inside the rapid transition region such as the minimum divergence. The conclusion seems to be that, when fully resolving the flow is either impossible or too costly, a "capturing" scheme such as ENO can be used on a coarse grid to obtain at least some partial information about the flow.

In this paper we perform a similar numerical study for the incompressible equation (1.1), to see what one can get by using the high-order ENO schemes on a coarse grid, without fully resolving the flow. We choose doubly periodic shear layers, used in [1], as our test case. A spectral method with $512^{2}$ points seems able to fully resolve the flow up to $t=8$, but begins to show signs of underresolution (wriggles in vorticity) thereafter. This indicates the tremendous requirement upon computation resources if one tries to resolve everything in the flow. We then use the third order ENO method (which is fourth order in the $L_{1}$ sense) and coarse grids $\left(64^{2}\right.$ and $128^{2}$ points), to assess its resolution. We find that the scheme remains stable for coarse meshes and certain quantities related to the smooth part of the flow, such as the total circulation around the roll-up, are adequately resolved by ENO methods.

A pioneer work in applying shock capturing compressible flow techniques to incompressible flow is by Bell, Colella and Glaz [1], in which they considered a second order Godunov type discretization, investigated the projection into divergence-free velocity fields for general boundary conditions, and discussed accuracy of time discretizations. Since our objective in this paper is to assess the resolution of the ENO method for (1.1), we choose a periodic boundary condition to simplify the projection. General boundary conditions would have to be handled either by more complicated projection [1] or by artificial compressibility methods [4]. We are currently investigating ENO schemes for such cases. 


\section{The ENO Method}

We solve (1.2) in its equivalent projection form

$$
\left(\begin{array}{l}
u \\
v
\end{array}\right)_{t}=\mathbf{P}\left[-\left(\begin{array}{c}
u^{2} \\
u v
\end{array}\right)_{x}-\left(\begin{array}{c}
u v \\
v^{2}
\end{array}\right)_{y}+\mu\left(\left(\begin{array}{c}
u \\
v
\end{array}\right)_{x x}+\left(\begin{array}{l}
u \\
v
\end{array}\right)_{y y}\right)\right]
$$

where $\mathbf{P}$ is the Hodge projection into divergence-free fields, i.e., if $\left(\begin{array}{c}\tilde{u} \\ \tilde{v}\end{array}\right)=\mathbf{P}\left(\begin{array}{l}u \\ v\end{array}\right)$, then $\tilde{u}_{x}+\tilde{v}_{y}=0$ and $\tilde{v}_{y}-\tilde{u}_{x}=v_{y}-u_{x}$. See, e.g., [1]. For the current periodic case the additional condition to obtain a unique projection $\mathbf{P}$ is that the mean values of $u$ and $v$ are preserved, i.e., $\int_{0}^{2 \pi} \int_{0}^{2 \pi} \tilde{u}(x, y) d x d y=\int_{0}^{2 \pi} \int_{0}^{2 \pi} u(x, y) d x d y$ and $\int_{0}^{2 \pi} \int_{0}^{2 \pi} \tilde{v}(x, y) d x d y=\int_{0}^{2 \pi} \int_{0}^{2 \pi} v(x, y) d x d y$.

We use $N_{x}$ and $N_{y}$ (even numbers) equally spaced grid points in $x$ and $y$, respectively. The grid sizes are denoted by $\Delta x=\frac{N_{x}}{2 \pi}$ and $\Delta y=\frac{N_{y}}{2 \pi}$, and the grid points are denoted by $x_{i}=i \Delta x$ and $y_{j}=j \Delta y$. The approximated numerical values of $u$ and $v$ at the grid point $\left(x_{i}, y_{j}\right)$ are denoted by $u_{i j}$ and $v_{i j}$.

We first describe the numerical implementation of the projection $\mathbf{P}$. In the periodic case this is easily achieved in the Fourier space. We first expand $u$ and $v$ using Fourier collocation:

$$
u_{N}(x, y)=\sum_{l=-\frac{N_{y}}{2}}^{\frac{N_{y}}{2}} \sum_{k=-\frac{N_{x}}{2}}^{\frac{N_{x}}{2}} \hat{u}_{k l} e^{I(k x+l y)}, \quad v_{N}(x, y)=\sum_{l=-\frac{N_{y}}{2}}^{\frac{N_{y}}{2}} \sum_{k=-\frac{N_{x}}{2}}^{\frac{N_{x}}{2}} \hat{v}_{k l} e^{I(k x+l y)}
$$

where $I=\sqrt{-1}, \hat{u}_{k l}$ and $\hat{v}_{k l}$ are the Fourier collocation coefficients which can be computed from the point values $u_{i j}$ and $v_{i j}$, using either FFT or matrix-vector multiplications. The detail can be found in, e.g., [3]. Derivatives, either by spectral method or by central differences, involve only multiplications by factors $d_{k}^{x}$ or $d_{l}^{y}$ in $(2.2)$ because $e^{I(k x+l y)}$ are eigenfunctions of such derivative operators. For example,

$$
d_{k}^{x}=I k, \quad d_{l}^{y}=I l
$$

for spectral derivatives;

$$
d_{k}^{x}=\frac{2 I \sin \left(\frac{k \Delta x}{2}\right)}{\Delta x}, \quad d_{l}^{y}=\frac{2 I \sin \left(\frac{l \Delta y}{2}\right)}{\Delta y}
$$

for the second order central differences which, when used twice, will produce the second order central difference approximation $\frac{w_{i+1}-2 w_{i}+w_{i-1}}{\Delta x^{2}}$ for $w_{x x}$, and

$$
d_{k}^{x}=\frac{2 I \sqrt{(1-\cos (k \Delta x))(7-\cos (k \Delta x))}}{\Delta x}, \quad d_{l}^{y}=\frac{2 I \sqrt{(1-\cos (l \Delta y))(7-\cos (l \Delta y))}}{\Delta y}
$$


for the fourth order central differences which, when used twice, will produce the fourth order central difference approximation $\frac{16\left(w_{i+1}+w_{i-1}\right)-\left(w_{i+2}+w_{i-2}\right)-30 w_{i}}{12 \Delta x^{2}}$ for $w_{x x}$. High order filters, such as the exponential filter [10], [9]:

$$
\sigma_{k}^{x}=e^{-\alpha\left(\frac{k}{N_{x}}\right)^{2 P}}, \quad \sigma_{l}^{y}=e^{-\alpha\left(\frac{l}{N_{y}}\right)^{2 P}}
$$

where $2 p$ is the order of the filter and $\alpha$ is chosen so that $e^{-\alpha}$ is machine zero, can be used to enhance the stability while keeping at least $2 p$-th order of accuracy. This is especially helpful when the projection $\mathbf{P}$ is used for the under-resolved coarse grid with ENO methods. We use the fourth order projection (2.5) and the filter (2.6) with $2 p=8$ in our calculations. This will guarantee third order accuracy (fourth order in $L_{1}$ ) of the ENO scheme. We will denote this combination (the fourth order projection plus the eighth order filtering) by $\mathbf{P}_{\mathbf{4}}$. To be precise, if $\left(\begin{array}{c}\tilde{u} \\ \tilde{v}\end{array}\right)=\mathbf{P}_{4}\left(\begin{array}{l}u \\ v\end{array}\right)$ and $\hat{u}_{k l}$ and $\hat{v}_{k l}$ are Fourier collocation coefficients of $u$ and $v$, then the Fourier collocation coefficients of $\tilde{u}$ and $\tilde{v}$ are given by

$$
\hat{\tilde{u}}=\sigma_{k}^{x} \sigma_{l}^{y} \frac{d_{l}^{y}\left(d_{l}^{y} \hat{u}-d_{k}^{x} \hat{v}\right)}{\left(d_{k}^{x}\right)^{2}+\left(d_{l}^{y}\right)^{2}}, \quad \hat{\tilde{v}}=\sigma_{k}^{x} \sigma_{l}^{y} \frac{-d_{k}^{x}\left(d_{l}^{y} \hat{u}-d_{k}^{x} \hat{v}\right)}{\left(d_{k}^{x}\right)^{2}+\left(d_{l}^{y}\right)^{2}}
$$

where $\sigma_{k}^{x}$ and $\sigma_{l}^{y}$ are defined by (2.6) with $2 p=8$, and $d_{k}^{x}$ and $d_{l}^{y}$ are defined by (2.5).

Next we shall describe the ENO scheme for (2.1). Since (2.1) is equivalent to the nonconservative form (1.1), it is natural to implement upwinding by the signs of $u$ and $v$, and to implement ENO equation by equation. The $r$-th order ENO approximation of, e.g., $\left(u^{2}\right)_{x}$ is thus summarized as follows:

1. Take $f(x)=u^{2}(x, y)$ with $y$ fixed. We start with the point values $f_{i}=f\left(x_{i}\right)$;

2. Define a function $h(x)$ satisfying $f(x)=\frac{1}{\Delta x} \int_{x-\frac{\Delta x}{2}}^{x+\frac{\Delta x}{2}} h(\xi) d \xi$, and its primitive $H(x)=$ $\int^{x} h(\xi) d \xi$. For each $j+\frac{1}{2}$, a $(r+1)$-th order polynomial $Q_{j+1 / 2}(x)$ is constructed interpolating $H(x)$ at points near $x_{j+1 / 2}$. It is remarked in [12] that the Newton divided differences of $H(x)$ are constant multiples of those of $f(x)$ of one order lower. Since we do not need the zeroth order difference, the approximation $Q_{j+1 / 2}$ of $H(x)$ can be accomplished using the information of $f_{i}$ only, without explicitly constructing $H(x)$ or its differences;

3. The stencil of the polynomial $Q(x)$ is determined adaptively by upwinding and smoothness of $f(x)$. It starts with either $x_{j}$ or $x_{j+1}$ according to whether $u \geq 0$ or $u<0$, then one point to the left or right is added to the stencil each time by comparing the two relevant divided differences and picking the smaller one in magnitude. 
4. The derivative $f^{\prime}(x)$ is finally approximated by the conservative difference $\frac{1}{\Delta x}\left(\hat{f}_{j+1 / 2}-\right.$ $\hat{f}_{j-1 / 2}$ ) where the numerical flux is computed by $\hat{f}_{j+1 / 2}=\left.\frac{d}{d x} Q(x)\right|_{x=x_{j+1 / 2}}$.

5. In the actual coding of the algorithm, undivided differences should be used instead of the divided differences to reduce round-off errors. There are also ways to make the procedure more economical on a vector computer. The details can be found in [13].

The approximation to $(u v)_{x}$ is the same as above with $f(x)=u(x, y) v(x, y)$, and that for $(u v)_{y}$ and for $\left(v^{2}\right)_{y}$ are similar, with upwinding based on $v$.

There are two ways to handle the second derivative terms for the Navier-Stokes equations. One can absorb them into the convection part and treat them using ENO. For example, $f(x)=u^{2}(x, y)$ can be replaced by $f(x)=u^{2}(x, y)-\mu u(x, y)_{x}$, where $u(x, y)_{x}$ itself can be obtained using either ENO or central difference of a suitable order. The remaining procedure for computing $f(x)_{x}$ would be the same as described above. Another simpler possibility is just to use standard central differences (of suitable order) to compute the double derivative terms. Our experience with compressible flow is that there is little difference between the two approaches, especially when the viscosity $\mu$ is small.

In the above we have described the discretization for the spatial derivatives

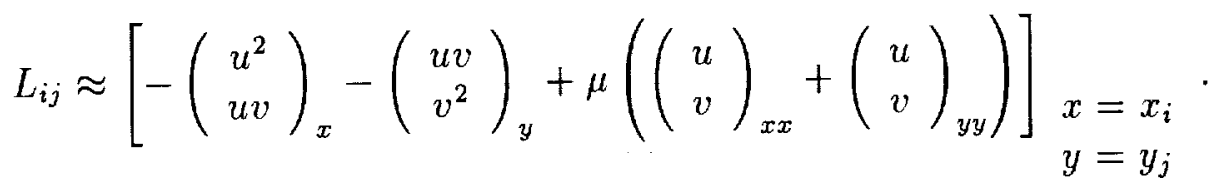

Next we use a third order TVD (total variation diminishing) Runge-Kutta method [11] to discretize the resulting ODE:

$$
\left(\begin{array}{l}
u \\
v
\end{array}\right)_{t}=\mathbf{P}_{4} L_{i j}
$$

obtaining:

$$
\begin{aligned}
& \left(\begin{array}{l}
u \\
v
\end{array}\right)^{(1)}=\mathbf{P}_{4}\left[\left(\begin{array}{l}
u \\
v
\end{array}\right)^{n}+\Delta t L_{i j}^{n}\right] \\
& \left(\begin{array}{l}
u \\
v
\end{array}\right)^{(2)}=\mathbf{P}_{4}\left[\frac{3}{4}\left(\begin{array}{l}
u \\
v
\end{array}\right)^{n}+\frac{1}{4}\left(\begin{array}{l}
u \\
v
\end{array}\right)^{(1)}+\frac{1}{4} \Delta t L_{i j}^{(1)}\right] \\
& \left(\begin{array}{l}
u \\
v
\end{array}\right)^{n+1}=\mathbf{P}_{4}\left[\frac{1}{3}\left(\begin{array}{l}
u \\
v
\end{array}\right)^{n}+\frac{2}{3}\left(\begin{array}{l}
u \\
v
\end{array}\right)^{(2)}+\frac{2}{3} \Delta t L_{i j}^{(2)}\right]
\end{aligned}
$$

Notice that we have used the property $P_{4} \circ P_{4}=P_{4}$ in obtaining the discretization (2.10) from $(2.9)$. 
This explicit time discretization is expected to be nonlinearly stable under the CFL condition

$$
\Delta t\left[\max _{i, j}\left(\frac{\left|u_{i j}\right|}{\Delta x}+\frac{\left|v_{i j}\right|}{\Delta y}\right)+2 \mu\left(\frac{1}{\Delta x^{2}}+\frac{1}{\Delta y^{2}}\right)\right] \leq 1
$$

(see [11]). For small $\mu$ (which is the case we are interested in) this is not a serious restriction on $\Delta t$.

\section{Numerical Results}

We present two numerical examples in this section.

Example 1: This example is used to check the third order accuracy of our ENO scheme for smooth solutions. We first take the initial condition as

$$
u(x, y, 0)=-\cos (x) \sin (y), \quad v(x, y, 0)=\sin (x) \cos (y)
$$

which was used in [5]. The exact solution for this case is known:

$$
u(x, y, t)=-\cos (x) \sin (y) e^{-2 \mu t}, \quad v(x, y, t)=\sin (x) \cos (y) e^{-2 \mu t}
$$

We take $\Delta x=\Delta y=\frac{1}{N}$ with $N=32,64,128$ and 256. The solution is computed up to $t=2$ and the $L_{2}$ error and numerical order of accuracy are listed in Table 3.1. For the $\mu=0.05$ case, we list results both with fourth order central approximation to the double derivative terms (central) and with ENO to handle the double derivative terms by absorbing them into the convection part (ENO). We can clearly observe fully third order accuracy (actually better in many cases because the spatial ENO is fourth order in the $L_{1}$ sense) in

\begin{tabular}{|c|c|c|c|c|c|c|}
\hline \multirow[t]{2}{*}{$\bar{N}$} & \multicolumn{2}{|c|}{$\mu=0$} & \multicolumn{2}{|c|}{$\mu=0.05$, central } & \multicolumn{2}{|c|}{$\mu=0.05$, ENO } \\
\hline & $L_{2}$ error & order & $L_{2}$ error & order & $\overline{L_{2} \text { error }}$ & order \\
\hline 32 & $9.10(-4)$ & & $5.28(-4)$ & & $4.87(-4)$ & \\
\hline 64 & $5.73(-5)$ & 3.99 & $3.20(-5)$ & 4.04 & $-5)$ & 3.98 \\
\hline 128 & $3.62(-6)$ & 3.98 & $1.93(-6)$ & 4.05 & $1.89(-6)$ & 4.03 \\
\hline 256 & $2.28(-7)$ & 3.99 & $1.18(-7)$ & 4.03 & $1.16(-7)$ & 4.03 \\
\hline
\end{tabular}
this table.

Table 3.1: Accuracy of ENO Schemes for (3.1)

We then take the initial condition as 


$$
u(x, y, 0)=-\sin ^{2}(x) \sin (2 y), \quad v(x, y, 0)=\sin (2 x) \sin ^{2}(y)
$$

which was used in [1]. We again take $\Delta x=\Delta y=\frac{1}{N}$ with $N=16,32,64,128$ and 256 and compute the solution up to $t=2$. However, this time the exact solution is unknown. As in [1], we test the accuracy by computing the $L_{2}$ difference between the solutions at the grid sizes $2 \Delta x$ and $\Delta x$, on the coarser grid. If $w_{\Delta x}=w+C \Delta x^{r}+O\left(\Delta x^{r+1}\right)$, then this difference $w_{2 \Delta x}-w_{\Delta x}=\left(2^{r}-1\right) C \Delta x^{r}+O\left(\Delta x^{r+1}\right)$ would predict the correct asymptotic order of accuracy $r$ and the error itself on the finer grid multiplied by $2^{r}-1$. The result is summarized in Table 3.2, where $\mathbf{w}=(u, v)^{T}$ and $L_{2} \operatorname{diff}=\left\|\mathbf{w}_{2 \Delta x}-\mathbf{w}_{\Delta x}\right\|_{L_{2}}$. In this table the estimated order and error are obtained using the remarks above. For $\mu=0.05$, we have again provided results both with fourth order central approximation to the double derivative terms (central) and with ENO to handle the double derivative terms by absorbing them into the convection part (ENO). The result in Table 3.2 reconfirms better than third order accuracy for the third order ENO scheme.

Table 3.2: Accuracy of ENO Schemes for (3.3)

\begin{tabular}{||r||r|r|r||r|r|r||r|r|r||}
\hline \hline \multicolumn{1}{|c||}{$N$} & \multicolumn{3}{|c||}{$\mu=0$} & \multicolumn{3}{c||}{$\mu=0.05$, central } & \multicolumn{3}{c||}{$\mu=0.05$, ENO } \\
\cline { 2 - 11 } & $L_{2}$ diff & order & error & $L_{2}$ diff & order & error & $L_{2}$ diff & order & error \\
\hline 32 & $1.14(-1)$ & & & $3.20(-2)$ & & & $3.60(-2)$ & & \\
\hline 64 & $1.40(-2)$ & 3.02 & $1.96(-3)$ & $2.78(-3)$ & 3.52 & $2.66(-4)$ & $2.93(-3)$ & 3.62 & $2.60(-4)$ \\
\hline 128 & $1.46(-3)$ & 3.26 & $1.69(-4)$ & $1.81(-4)$ & 3.94 & $1.26(-5)$ & $1.80(-4)$ & 4.02 & $1.18(-5)$ \\
\hline 256 & $1.11(-4)$ & 3.77 & $8.78(-6)$ & $1.09(-5)$ & 4.06 & $6.91(-7)$ & $1.10(-5)$ & 4.04 & $7.15(-7)$ \\
\hline \hline
\end{tabular}

Example 2: This is our test example to study resolution of ENO schemes when the grid is coarse. It is a double shear layer taken from [1]:

$$
u(x, y, 0)=\left\{\begin{array}{ll}
\tanh ((y-\pi / 2) / \rho) & y \leq \pi \\
\tanh ((3 \pi / 2-y) / \rho) & y>\pi
\end{array} \quad v(x, y, 0)=\delta \sin (x)\right.
$$

where we take $\rho=\pi / 15$ and $\delta=0.05$. The Euler equations $(\mu=0)$ are used for this example. The solution quickly develops into roll-ups with smaller and smaller scales, so on any fixed grid the full resolution is lost eventually. For example, the expensive run we performed using $512^{2}$ points for the spectral collocation code (with a 18-th order filter (2.4)) seems able to resolve the solution fully up to $t=8$, then begins to lose resolution as indicated by the wriggles in the vorticity contour at $t=10$ (the last contour in Fig. 1). On the other hand, the ENO runs with $64^{2}$ and $128^{2}$ points produces smooth, stable results (Fig. 2 and 3). Apparently with these coarse grids the full structure of the roll-up is not resolved. However, when we compute the total circulation 


$$
c_{\Omega}=\int_{\Omega} \omega(x, y) d x d y=\int_{\partial \Omega} u d x+v d y
$$

around the roll-up by taking $\Omega=\left[\frac{\pi}{2}, \frac{3 \pi}{2}\right] \times[0,2 \pi]$ and using the rectangular rule (which is infinite order accurate for the periodic case) on the line integrals at the right-hand-side of (3.5), we can see that this number is resolved much better than the roll-up itself (see Table $3.3)$.

Table 3.3: Resolution of the Total Circulation

\begin{tabular}{|c|c|c|c|c|c|}
\hline$t$ & 2 & 4 & 6 & 8 & 10 \\
\hline ENO $64^{2}$ & 0.87300 & 3.07100 & 7.16889 & 9.88063 & 10.90122 \\
\hline ENO $128^{2}$ & 0.87452 & 2.97810 & 7.30999 & 10.34414 & 11.79418 \\
\hline spectral $512^{2}$ & 0.87433 & 2.98029 & 7.28308 & 10.46212 & 11.85875 \\
\hline
\end{tabular}

\section{Concluding Remarks}

High order ENO schemes can be applied to the incompressible Euler and Navier-Stokes equations to obtain stable, under-resolved results on a coarse grid. Some global quantities such as the circulation around the roll-up region can be faithfully resolved. 


\section{References}

[1] J. Bell, P. Colella and H. Glaz, A Second Order Projection Method for the Incompressible Navier-Stokes Equations, J. Comput. Phys., v85, 1989, pp.257-283.

[2] P. Colella and P. Woodward, The Piecewise Parabolic Method (PPM) for GasDynamical Simulations, J. Comput. Phys., v54, 1984, pp.174-201.

[3] C. Canuto, M.Y. Hussaini, A. Quarteroni and T. A. Zang, Spectral Methods in Fluid Dynamics, Springer-Verlag, 1988.

[4] A. Chorin, A Numerical Method for Solving Incompressible Viscous Flow Problems, J. Comput. Phys., v2, 1967, pp.12-26.

[5] A. Chorin, Numerical Solution of the Navier-Stokes Equations, Math. Comput., v22, 1968, pp.745-762.

[6] D. Gottlieb and S. Orszag, Numerical Analysis of Spectral Methods: Theory and Applications, v26, SIAM-CBMS, Philadephia, 1977.

[7] A. Harten, High Resolution Schemes for Hyperbolic Conservation Laws, J. Comput. Phys., v49, 1983, pp.357-393.

[8] A. Harten, B. Engquist, S. Osher and S. Chakravarthy, Uniformly High Order Accurate Essentially Non-Oscillatory Schemes, III, J. Comput. Phys., v71, 1987, pp.231-303.

[9] D. A. Kopriva, A Practical Assessment of Spectral Accuracy for Hyperbolic Problems with Discontinuities, J. Sci. Comput., v2, 1987, pp.249-262.

[10] A. Majda, J. McDonough and S. Osher, The Fourier Method for Nonsmooth Initial Data, Math. Comput., v32, 1978, pp.1041-1081.

[11] C.-W. Shu and S. Osher, Efficient Implementation of Essentially Non-Oscillatory Shock Capturing Scheme, J. Comput. Phys., v77, 1988, pp.439-471.

[12] C.-W. Shu and S. Osher, Efficient Implementation of Essentially Non-Oscillatory Shock Capturing Scheme, II, J. Comput. Phys., v83, 1989, pp.32-78.

[13] C.-W. Shu, T.A. Zang, G. Erlebacher, D. Whitaker and S. Osher, High Order ENO Schemes Applied to Two- and Three-Dimensional Compressible Flow, Appl. Numer. Math., v9, 1992, pp.45-71. 
Figure 1: The vorticity contour for Example 2 at $t=4,6,8,10$. Spectral method with $512^{2}$ points.
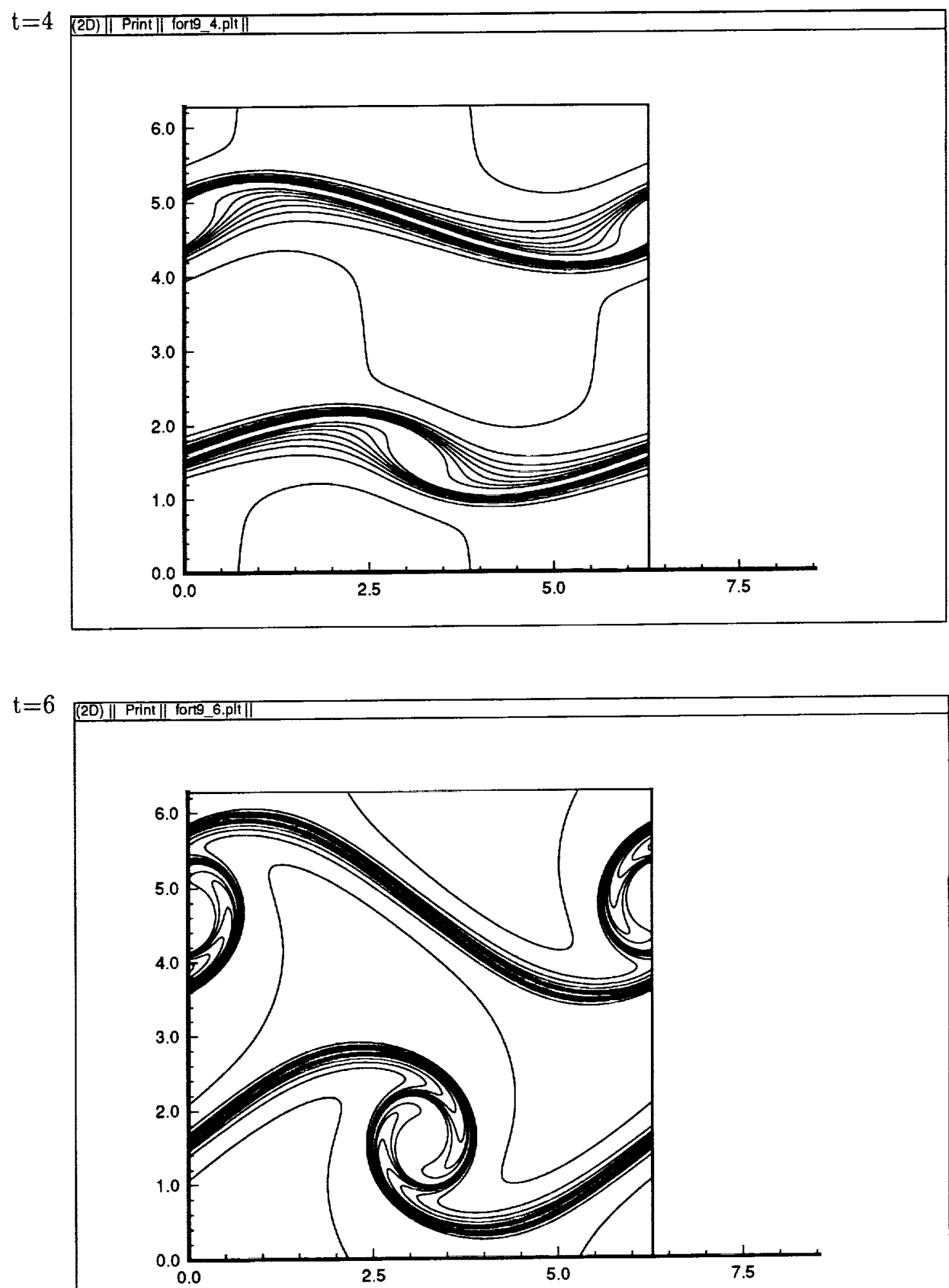
Figure 1 continued:
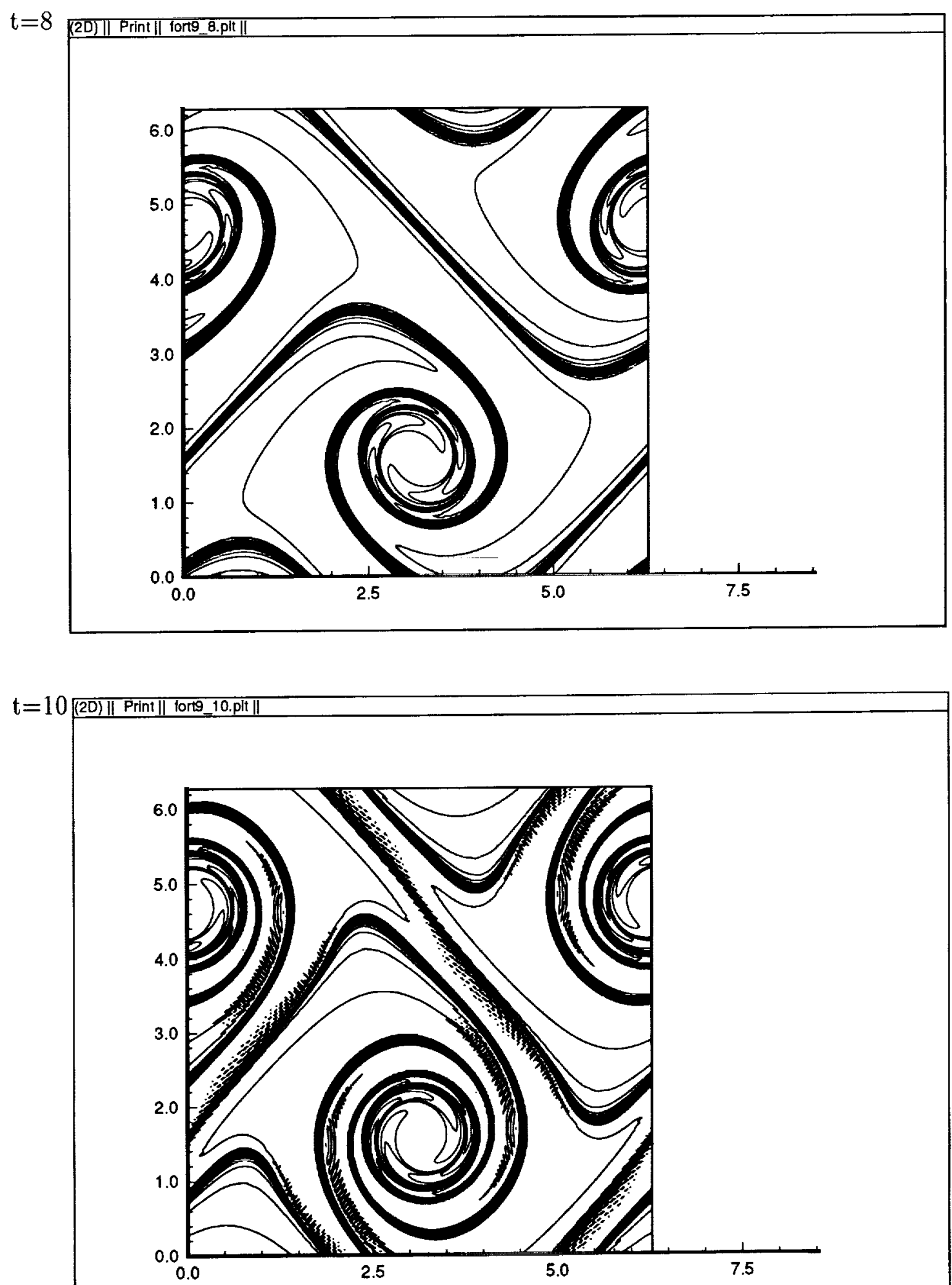
Figure 2: The vorticity contour for Example 2 at $t=4,6,8,10$. ENO method with $64^{2}$ points.
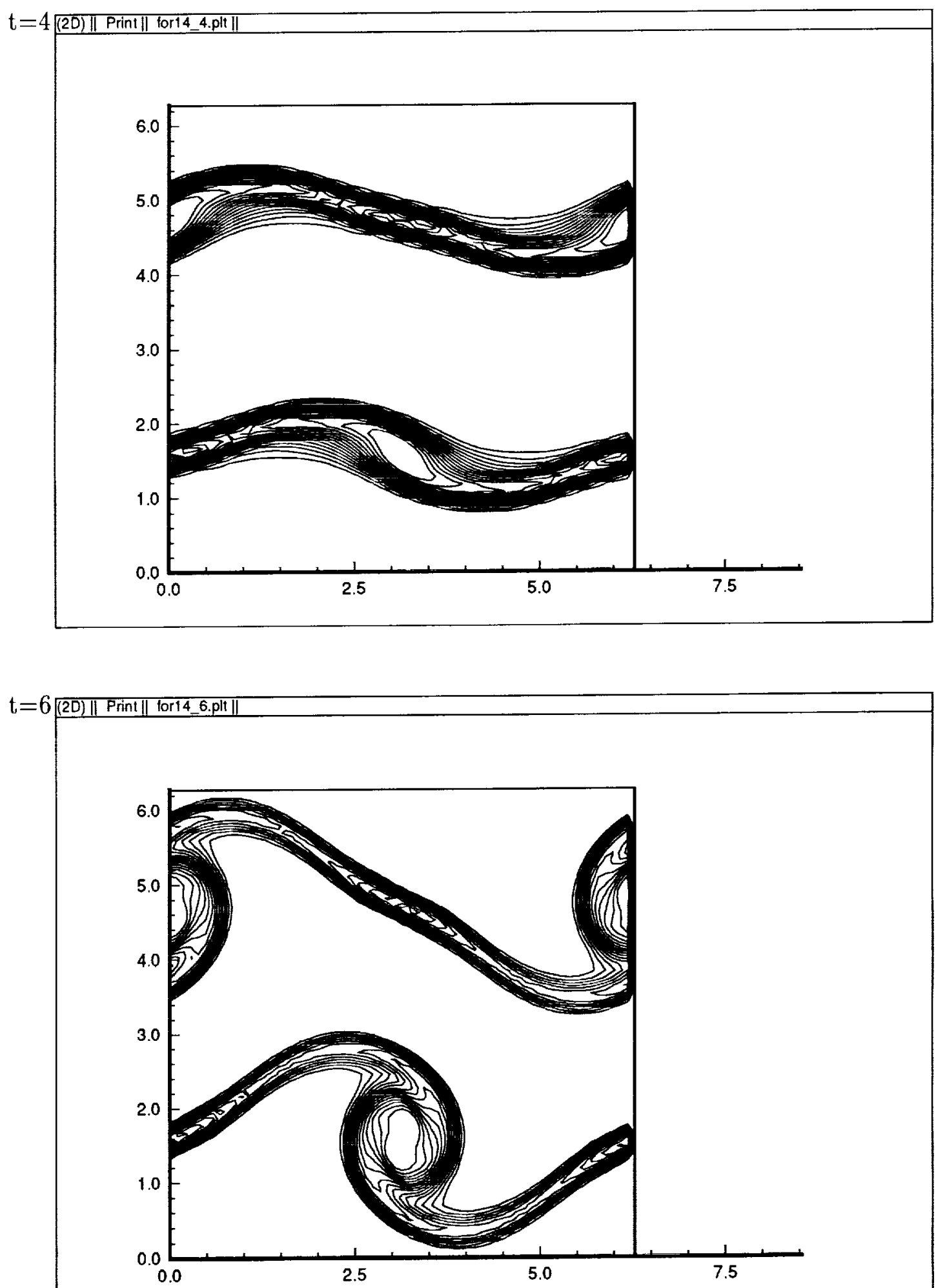
Figure 2 continued:
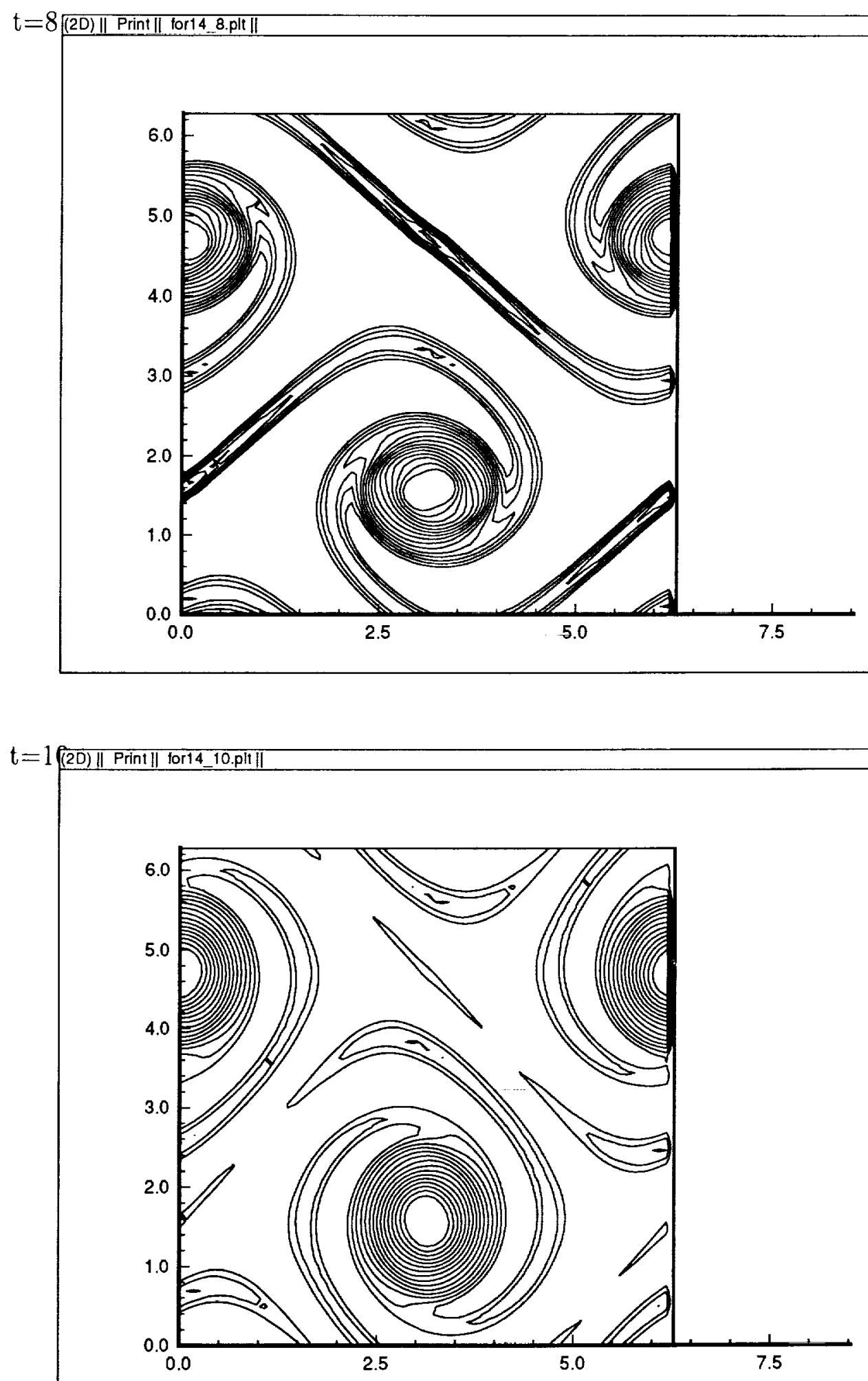
Figure 3: The vorticity contour for Example 2 at $t=4,6,8,10$. ENO method with $128^{2}$ points.
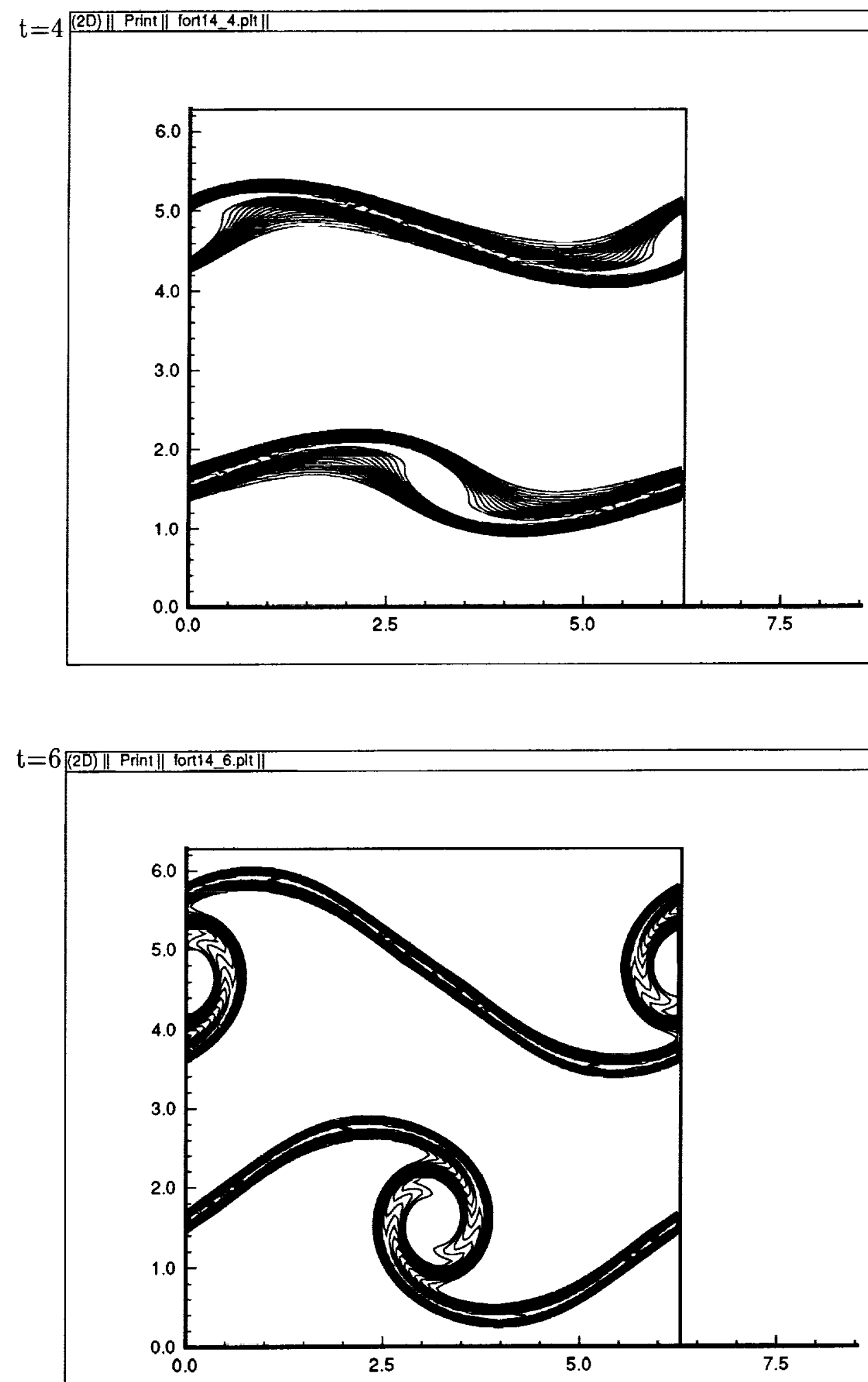
Figure 3 continued:
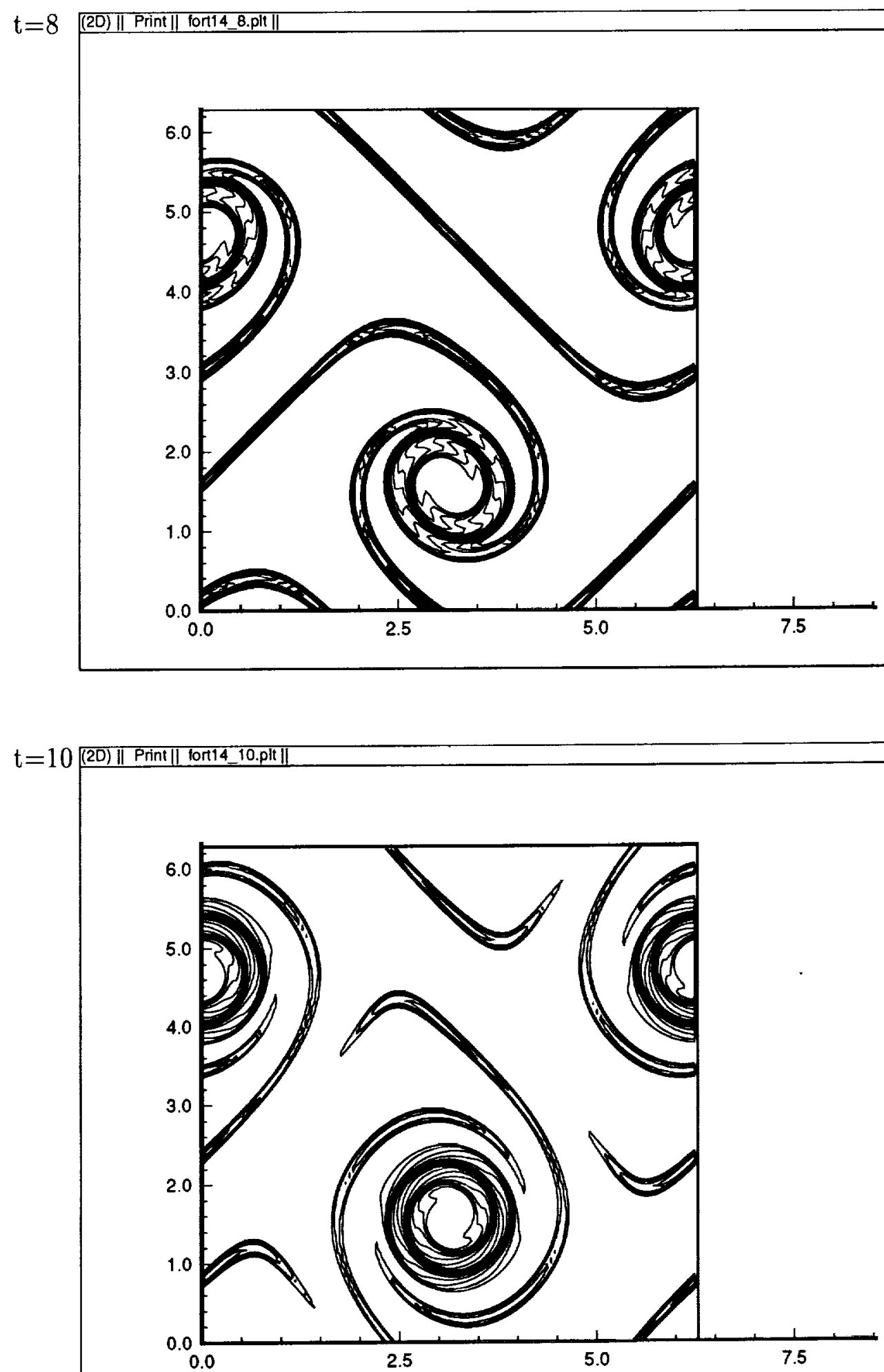



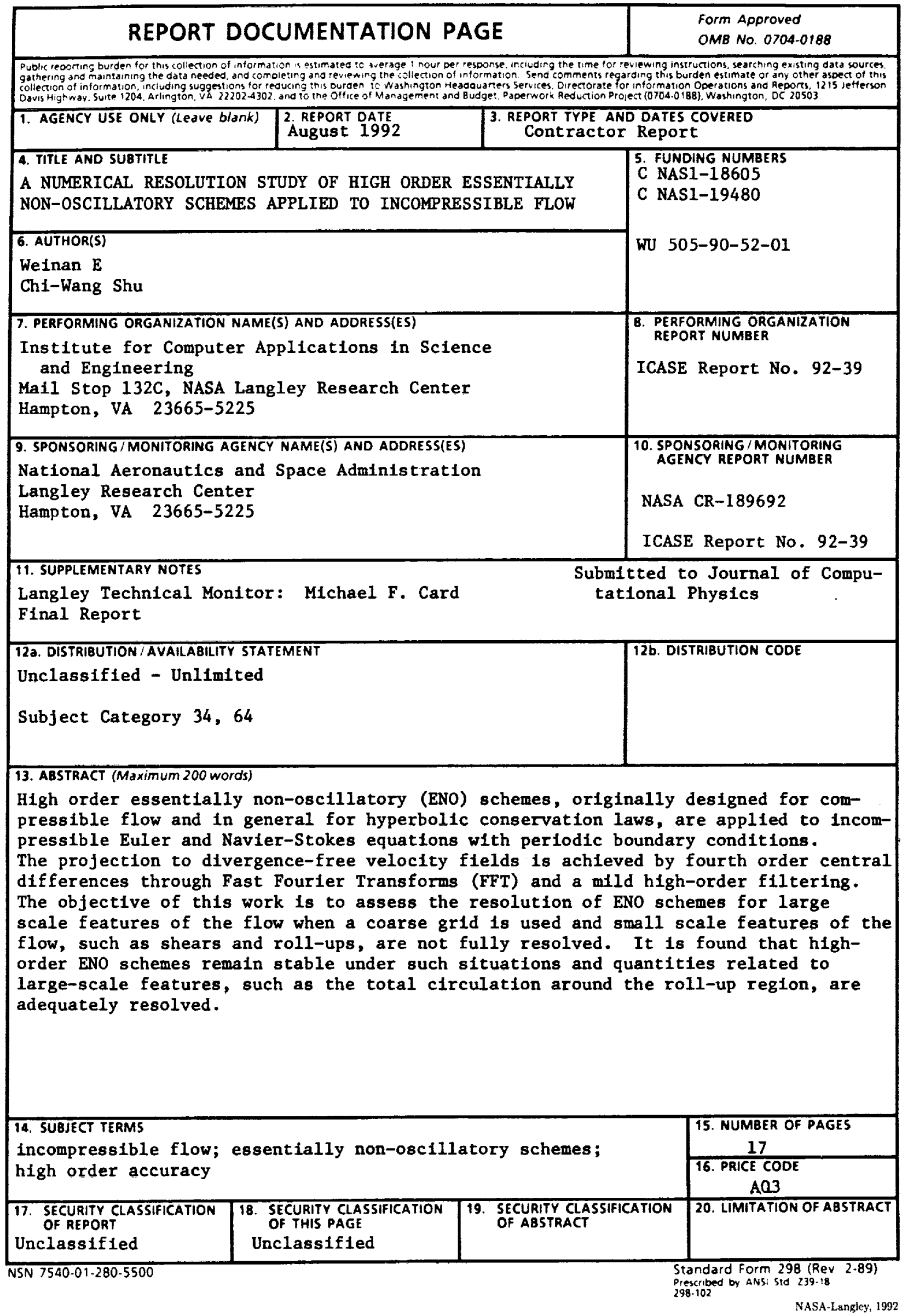

